\title{
Development of the Two D Wall for Simulation of Glint from Atmospheric Propagation and Multipath ${ }^{1,2}$
}

\author{
Robert Penno \\ Department of Electrical \& Computer Engineering \\ University of Dayton, Dayton, OH 45429-0226, \\ (937) 229 3984, \\ robert.penno@notes.udayton.edu
Seng Hong, William Austin, John Glett, Gwynne Jones
Sensors Directorate, Air Force Research Lab,
WPAFB, OH 45433, (937) 255 6506, ext 3617
seng.hong@wpafb.af.mil,
william.austin@wpafb.af.mil, john.glett@wpafb.af.mil
Mark Haenni
MacAulay-Brown, Inc., Dayton, OH 45433
mark.haenni@wpafb.af.mil, gwynne.jones@wpafb.af.mil
Rey Febo, University of Puerto Rico, Mayaguez, PR 00681,rfs14879@uprm.edu

\begin{abstract}
In the development of airborne radars, it is often useful to have a means of testing and evaluating the efficacy of the radar in the presence of angle (or phase) noise, referred to as glint. Glint typically occurs when the illuminated body is characterized by multiple scattering centers whose relative positions shift while the object is being illuminated. These phase shifts produce a distorted return which can undermine the accurate determination of the attributes of the object such as location or velocity. In addition to this source of phase noise, other sources of phase noise include atmospheric scintillation as well as reflections of a scattered return from adjacent obstacles, (i.e. multipath). The ability to evaluate the effects of the sources of phase noise is highly useful in developing methods to mitigate their effects. The "Two D Wall" would provide such an environment.
\end{abstract}

\section{TABLE OF CONTENTS}

1. INTRODUCTION.....................................................1

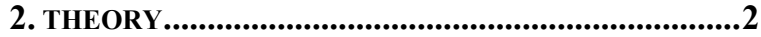

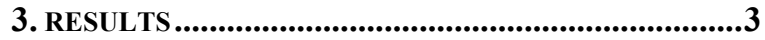

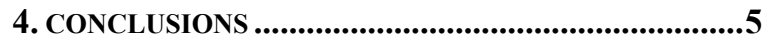

REFERENCES ............................................................66

ACKNOWLEDGEMENT................................................6

BIOGRAPHY ..........................................................6

1 "U.S. Government work not protected by U.S. copyright."

${ }^{2}$ IEEEAC paper\#1095, Version 3, Updated December 17, 2007

\section{INTRODUCTION}

The notion of a triad antenna array was first described by Hong \& Kissell [1] when they demonstrated the effectiveness of a single three-element array, a triad, in generating a glinted signal. This work was originally pursued to develop methods of improving monopulse radar systems in the presence of glinted scatterers. Such systems respond to the plane of the incoming phase front of a scatterer, causing the plane of the array to re-orient itself so that it is normal to that incoming phase plane. If that incident phase front is glinted, the resulting angular error in the phase front can cause the tracking radar to lose track of the scatterer. Their paper described how a target with angular error in its radar return could be generated, and the phase plane incident upon the radar could be depicted. In their work, the spacing of the three elements in the array, as well as the separation of the array from the receiver aperture

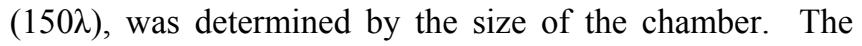
work described extends results by showing the effect of the addition of a prescribed amount of phase noise to both stationary and moving objects. Furthermore, results are shown for a moving object simulated by a multiple triad array, a precursor to the development of a much larger "Two D Wall", comprised of several hundred antenna elements, arranged in a triangular array, capable of 
simulating four (or six) different objects.

Experimental results, in conjunction with computer modeling, will be displayed to demonstrate the effects of glint, or angle scintillation, on the radar return of a complex object. As a prototype, this "Two D Wall" will serve to guide a development for a much larger version to include more than 200 antenna elements and four (to six) signal generators. The challenges of this larger scale "Two D Wall" include development of multiple port power combiners, dividers and RF switches that provide signal paths with acceptable power losses. New technologies, such as metamaterials, are being explored for this task.

\section{THEORY}

The fundamental component of this work is the three element array, referred to as a triad, shown in Figure 1. Intrinsic to this array is the property of a triangular geometry that

$$
a+b+c=L(\sqrt{3}) / 2
$$

When normalized by the height of the triad, this becomes

$$
a_{n}+b_{n}+c_{n}=1,
$$

If the radiated power from each element in the array is regulated by this condition, then the composite signal in the far field is perceived by the receiver as being located at some point interior to the triad. Thus,

$$
V_{a o o} e^{-j \beta R_{1}}+V_{b o o} e^{-j \beta R_{2}}+V_{c o o} e^{-j \beta R_{3}}=E_{r e c}
$$

Where the strengths of the fields, $\mathrm{V}_{\mathrm{i}}$, radiated from the triad are complex, i.e. having magnitude and phase, defined by

$$
\begin{aligned}
& V_{\text {aoo }}=a_{n} e^{j \psi_{a}}, \\
& V_{\text {boo }}=b_{n} e^{j \psi_{b}}, \text { and } \\
& V_{\text {coo }}=c_{n} e^{j \psi_{c}}
\end{aligned}
$$

A receiver, placed in the far field along the $+Z$-axis, perceives a single, complex scatterer whose phase center is located at the point interior to the array defined by the magnitudes, $V_{i}$. Simulation of a moving target is accomplished by changing of the magnitudes, $\mathrm{V}_{\mathrm{i}}$, (and, hence, the triple $\left.\left(a_{n}, b_{n}, c_{n}\right)\right)$ while changes in the phase of $\mathrm{V}_{\mathrm{i}}$ are perceived by the receiver as glint.

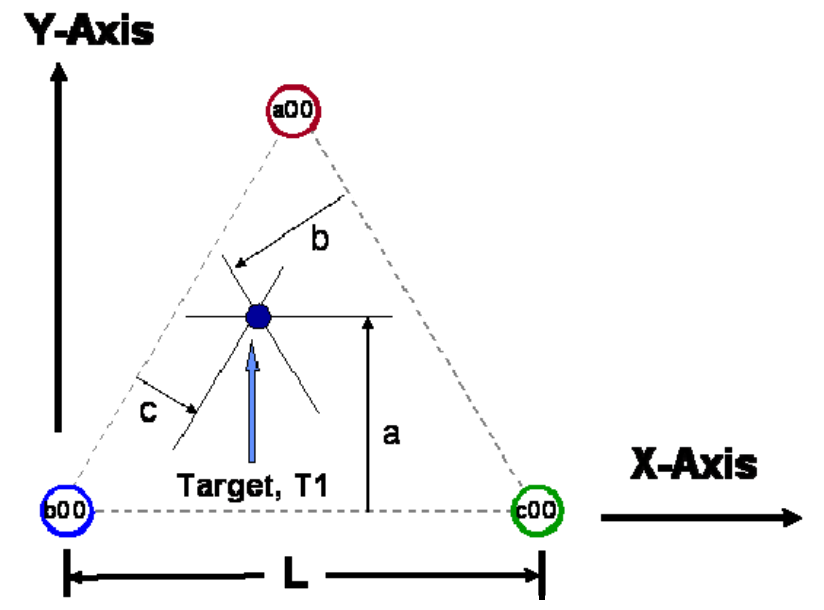

Figure 1: A Triangular Array

To implement a multiple triad array that simulates multiple scatterers, or a single scatterer and its multipath image, a six element triangular array can be implemented. A schematic of such a system is shown in figure 2, which includes two signal generators and six antennas arranged in four triads. With each signal generator is associated an amplifier and three vector modulators, each with separate control of attenuation and phase.

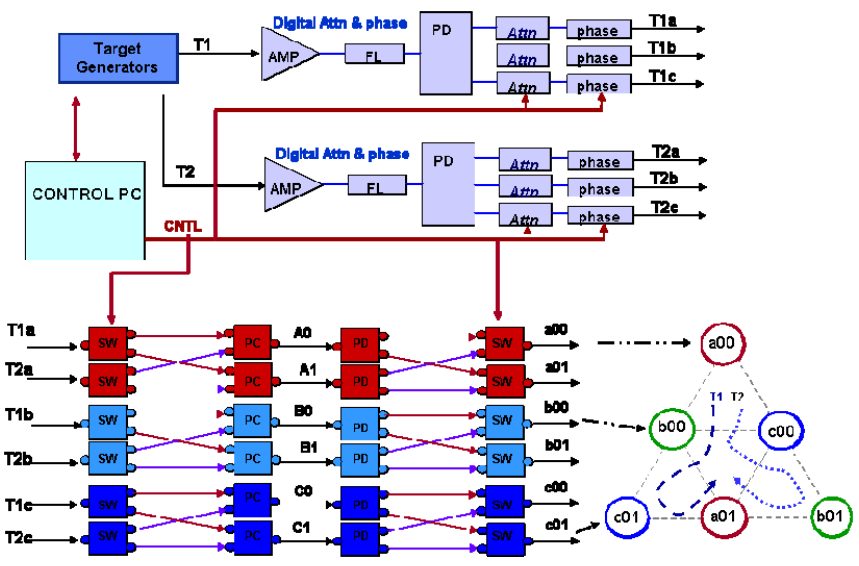

Figure 2: Two Source, Six Element Configuration

The output of each signal generator is power divided into three separate vector modulators, where the values of $\mathrm{V}_{\text {aoo }}$, $\mathrm{V}_{\text {boo }}$ and $\mathrm{V}_{\text {coo }}$ are set as prescribed by equations (3) and (4) These outputs are, in turn, submitted to the matrix of switches, power combiners and power dividers which control the triad to which each signal is directed. A pc is used to affect such control. The amplifiers placed at the output of the signal generators, ahead of the vector modulators, are used to affect the perceived range of the scatterer from the receiver. Thus, a single generator, $T_{1}$, could be fed into a two-way power divider (ahead of the amplifier) into path $T_{1}$, with the other output fed via a phase shifter to path $T_{2}$, to implement a multipath signal. 


\section{RESULTS}

To test the validity of these ideas, a six-element array was constructed as shown in figure 3, using Vivaldi horn antennas as the radiating elements arranged in a triangular array with 15" sides. In all testing, a combination gain, phase comparison monopulse receiver was used to detect the received signal, while the single signal generator operated at $9.7 \mathrm{GHz}$.

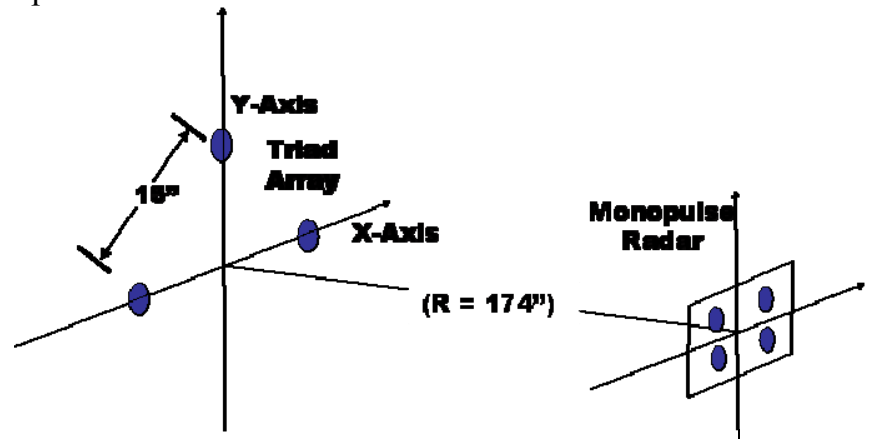

Figure 3: Test Setup

Figure 4 shows the six-element array as it was constructed. In the results that follow, the single triad refers to the top triad of the six element array shown in this figure.

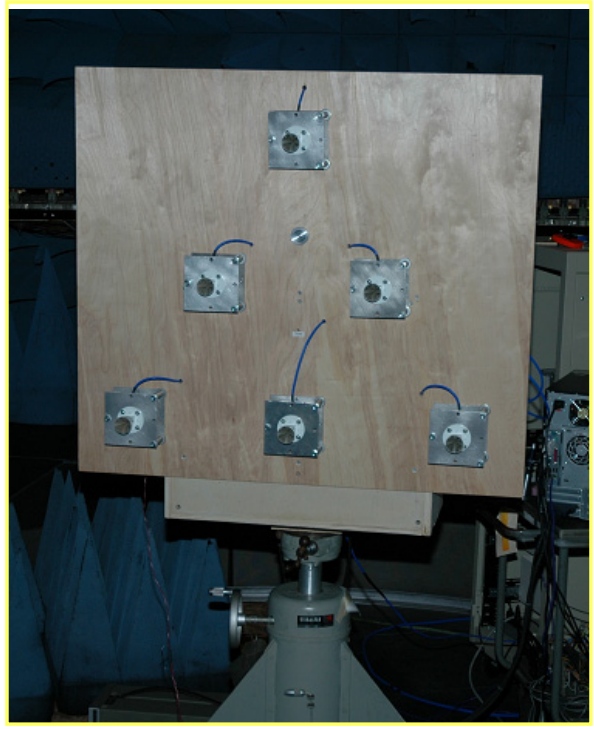

Figure 4: The Six Element, Four Triad Array

In the first test performed, a single triad was energized to produce a single scatterer at the centroid of the triad. Then uniform phase noise was a zero-mean, uniform random variable added to the phase component of each of the three vector modulators, and was characterized by the range of the phase variation. Shown in figure 5 is the intended radiation pattern outlined against the location of the three horn antennas, located at the vertices of the triad, while figure 6 shows the scattering center as perceived by the monopulse receiver, located $142.9 \lambda$ away. No phase noise has been added.

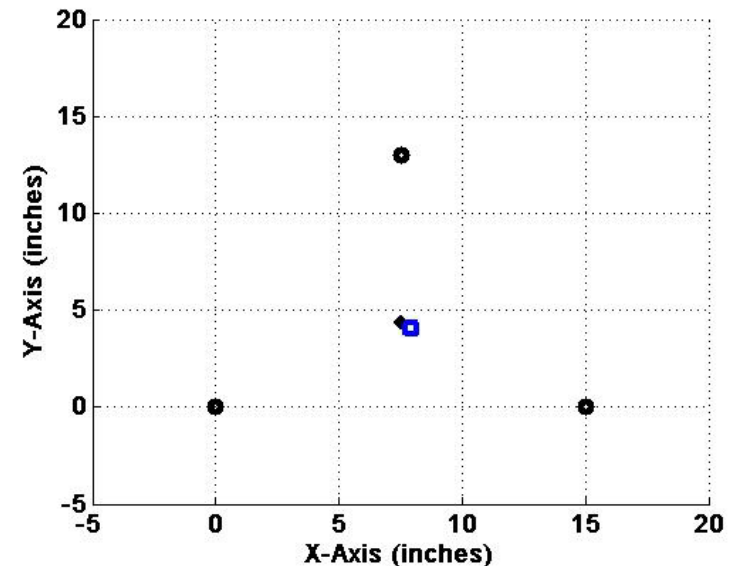

Figure 5: Intended Scattering Center

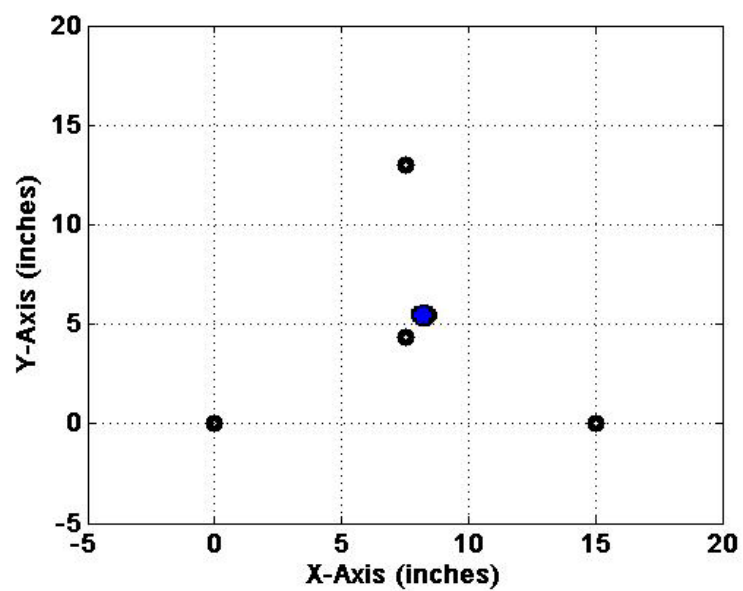

Figure 6: Perceived Scattering Center

The addition of noise $\left(-10^{\circ} \leq \phi_{\mathrm{n}} \leq 10^{\circ}\right)$ to all three phases, produces very little effect in the receiver's ability to discern the location of the scatterer, as is shown in figure 7

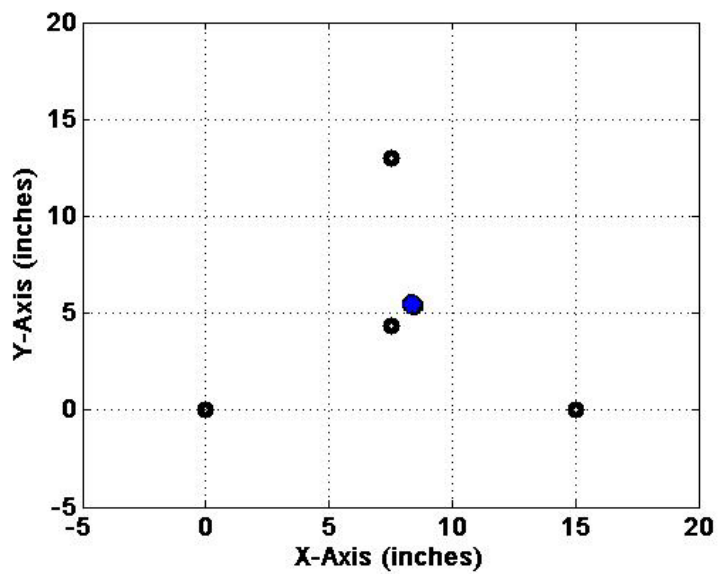

Figure 7: Receiver Estimate with the Addition of $10^{\circ}$ Phase Noise to all Radiators

Results of addition of more phase noise $\left(-30^{\circ} \leq \phi_{\mathrm{n}} \leq 30^{\circ}\right)$ exclusively to a single radiator located at the top of the triad, is shown in Figure 8. 


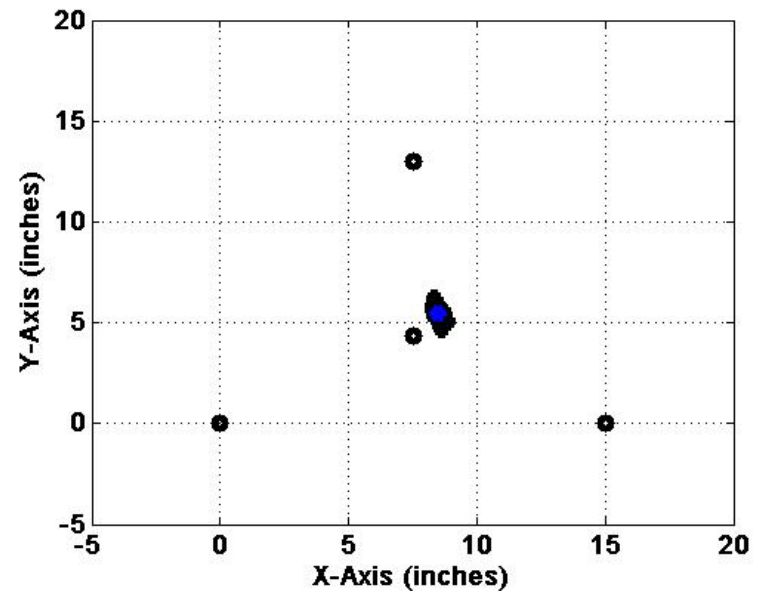

Figure 8: Receiver Estimate with the Addition of $30^{\circ}$ Phase Noise the Top Radiator

The addition of this same phase noise to all three radiators simultaneously is shown in figure 9.

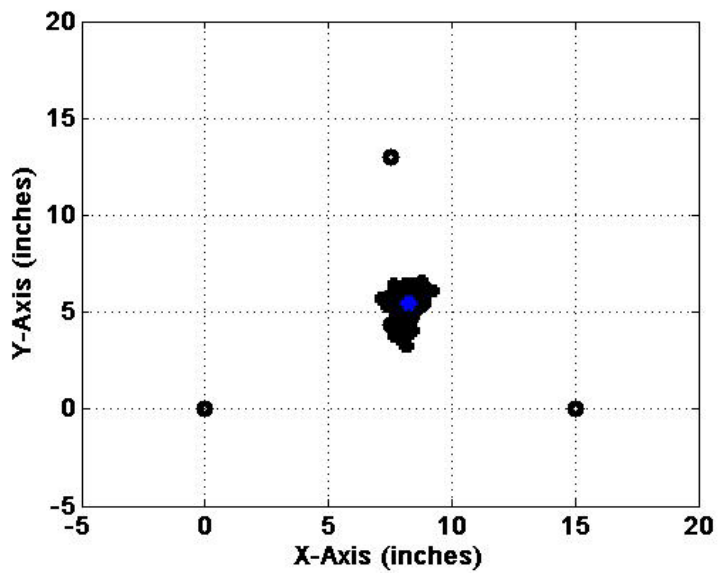

Figure 9: Receiver Estimate with the Addition of $30^{\circ}$ Phase Noise All Three Radiators

It is seen that the receiver location estimates are beginning to diverge significantly. However, the mean estimate is accurate. Similarly, Figure 10 demonstrates an ever widening plot of estimates, with the mean estimate (shown in blue) remaining true to the actual location.

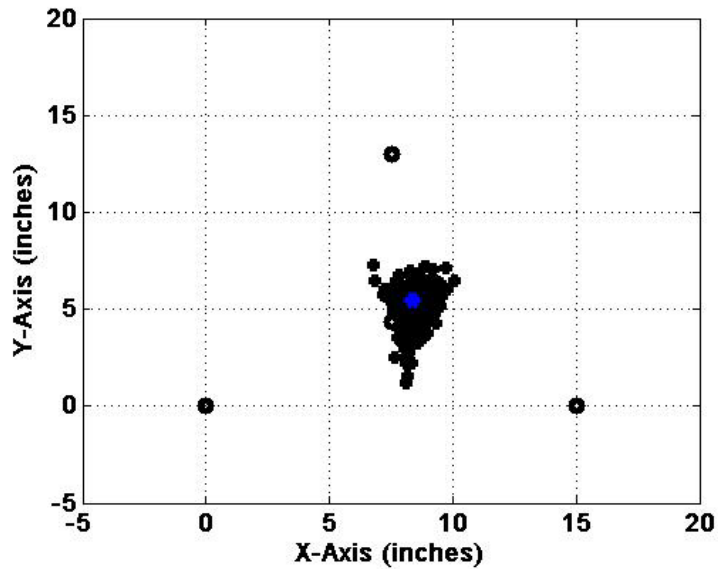

Figure 10: Receiver Estimate with the Addition of $40^{\circ}$ Phase Noise All Three Radiators

The threshold is breached with the addition of phase noise to all three receivers of $\left(-52^{\circ} \leq \phi_{n} \leq 52^{\circ}\right)$. In Figure 11 it is seen that the mean estimate (shown in blue with an arrow) is shifted from the centroid of the triad.

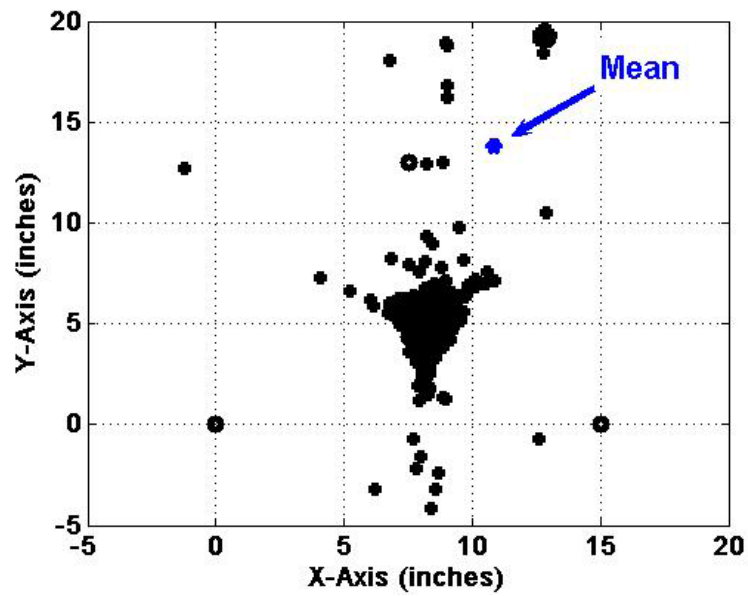

Figure 11: Receiver Estimate with the Addition of $52^{\circ}$ Phase Noise All Three Radiators

Further increases of phase noise saw a movement of the mean estimate out of the triad completely.

The ability to simulate a moving source was next evaluated. Again using the single triad with a single source, a raster path was simulated. As shown in Figure 12, this raster path began near the lower left element and progresses upward to the top of the triad. Figure 12 shows the intended path of the simulated source movement, the radiated path and the path estimated by the monopulse receiver. The radiated path is a plot of the complex sum of the signals produced by the three vector modulators and includes any phase noise that is added. In Figure 12, no phase noise has been added.

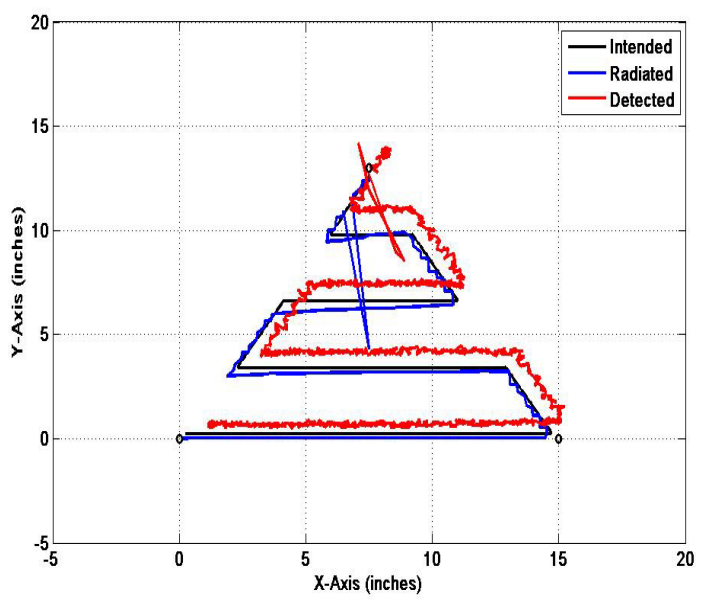

Figure 12: Receiver Estimate with No Phase Noise

The addition of phase noise $\left(-10^{\circ} \leq \phi_{n} \leq 10^{\circ}\right)$ to all three 
phases is shown in Figures 13 and phase noise $\left(-20^{\circ} \leq \phi_{\mathrm{n}} \leq\right.$ $20^{\circ}$ ) to all three phases is shown in Figure 14. It is seen that the distortion of the receiver estimates increases with phase noise, or simulated glint.

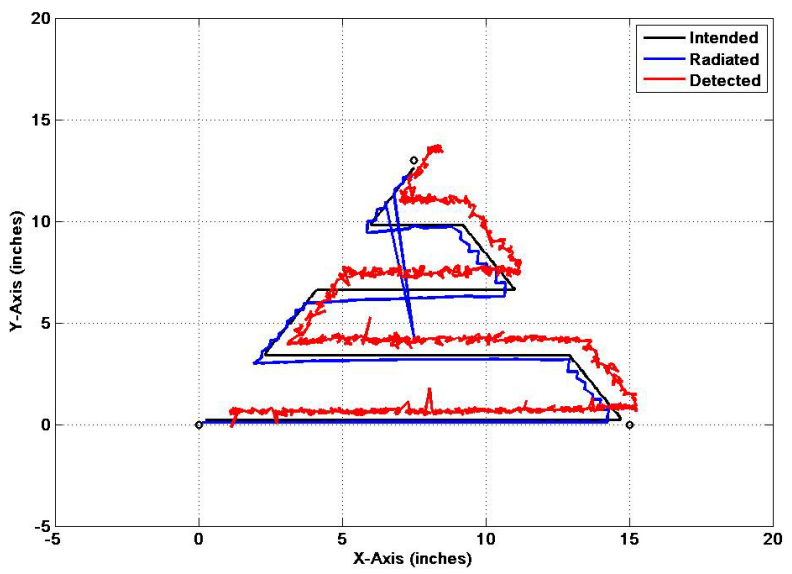

Figure 13: Receiver Estimate with the Addition of $10^{\circ}$ Phase Noise All Three Radiators

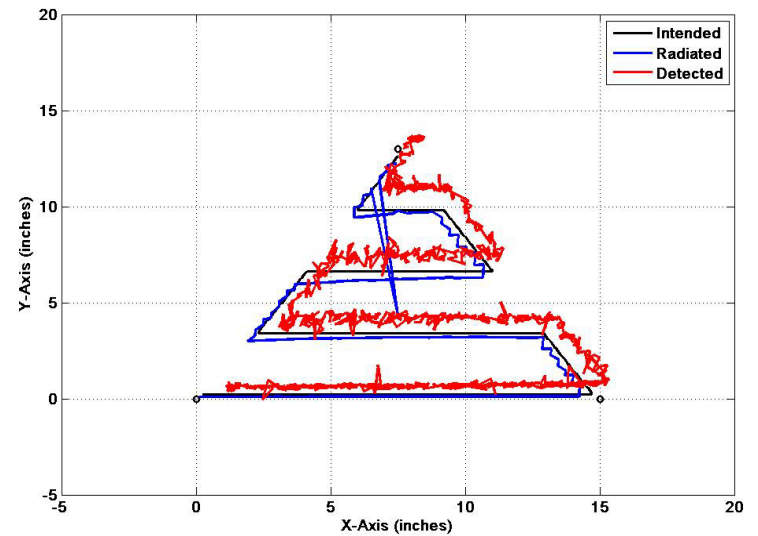

Figure 14: Receiver Estimate with the Addition of $20^{\circ}$ Phase Noise All Three Radiators

Finally, Figure 15, shows increased distortion in the location estimates of the receiver when the phase noise ($30^{\circ} \leq \phi_{\mathrm{n}} \leq 30^{\circ}$ ) is increased.

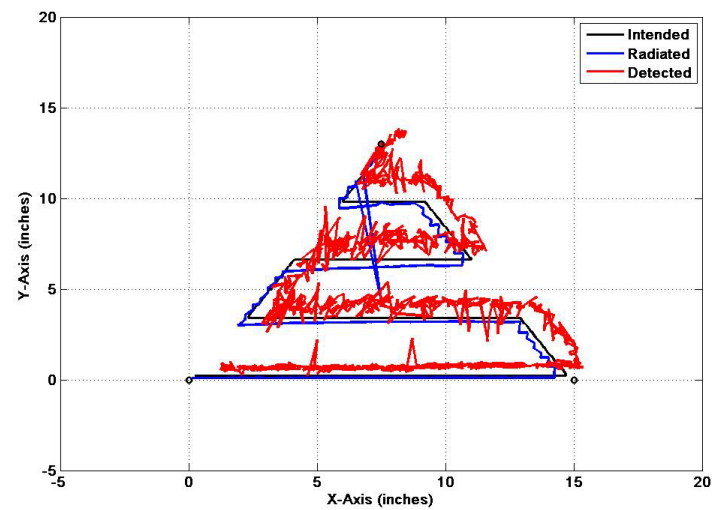

Figure 15: Receiver Estimate with the Addition of $30^{\circ}$ Phase Noise All Three Radiators
In Figures (12)-(15), the code used to generate the path radiated by the six-element array included a point outside of the four triads. When this happened, the default point at the centroid was radiated. Hence, the anomalous point in these figures is shown, along with the location estimated by the receiver.

The next level of complexity was to implement a moving path over the entire six-element array. Again, a raster path was chosen. This path in particular challenges the ability of the array to properly select the location of the correct triad from which to radiate the simulated signal. Figure 16 shows the intended path against the path as estimated by the monopulse receiver. The locations of the six elements, defining the four triads, are also shown. The location estimates from the receiver mirror with reasonable accuracy the simulated movement of the source.

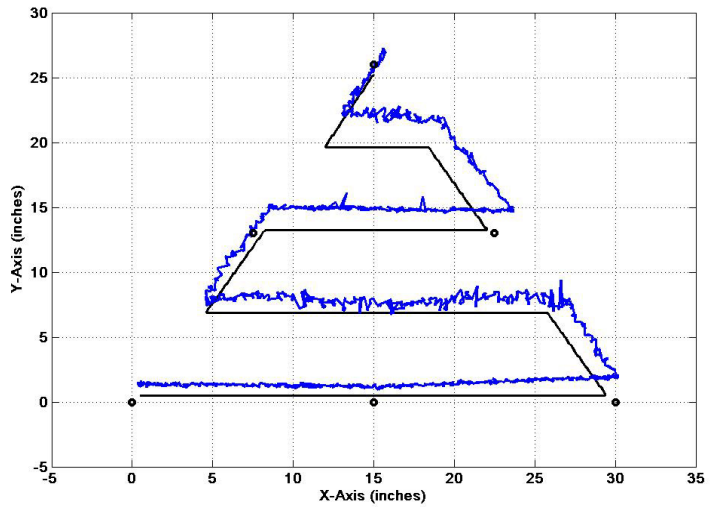

Figure 16: Receiver Estimate with the Addition of $20^{\circ}$ Phase Noise All Three Radiators

\section{CONCLUSIONS}

It has been shown that the six elements, triangular array with proper switching can simulate a complex scatterer inside a chamber to a typical radar receiver. Adjustments to the phase of the radiated signals at properly chosen elements are perceived by the receiver as glinted radar returns, which can be used to develop techniques to mitigate against such glint. It was demonstrated that this array can be used to simulate a signal to test a receiver to its threshold of being able to provide accurate location estimates. Furthermore, it was demonstrated that this array can accurately simulate a moving source modeled by a complex radar return.

The array has several, as yet, unrealized capabilities. The ability to model the multipath return of a single source is within the scope of this array, and will be realized when a second source is implemented. A much larger array, comprised of several hundred elements, is envisioned and four (or even six) sources is envisioned. Such an array, the "Two D Wall", would require significant microwave components, such as 70-way power dividers, that are typically characterized by high losses. An analysis 
of such a signal path revealed that $30 \mathrm{~dB}$ of loss per source path to an antenna was not unreasonable for such an expanded array. Research is ongoing into the application of new concepts, such as metamaterials [2], to reduce these losses. Moreover, the "Two D Wall" has the potential to provide a test bed in which many applications may evaluate and develop techniques to deal with glint, or glint-related phenomena.

\section{REFERENCES}

[1] "Emulate Glint Signal with a Simple Triad Antenna Array”, S. M. Hong, R. M Kissell, RF Design, September 1998

[2]"A Metamaterial T-Junction Power Divider", E. Saenz et al, IEEE Microwave and Wireless Components Letters, Vol. 17, No3, March 2007, pp172-174

\section{ACKNOWLEDGEMENT}

We would like to thanks the RCA Lab team lead, Capt. Jason Ives for his support, and Mr. Alex Dudash, Mr. Walter Joynes, Mr. Richard Myers for data collection efforts. Our appreciation goes to Ms. Sharon Williamson for her proof read, and the RF Sensor Assessment branch chief, Mr. Tracy Johnston, for funding this work in part.

\section{BIOGRAPHY}

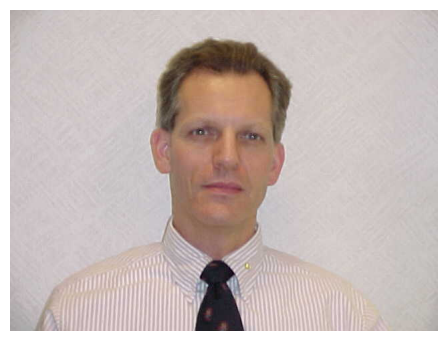

Dr. Robert Penno is an Associate Professor in the Department of Electrical and Computer Engineering at the University of Dayton, Dayton, Ohio. He received a B.S.M.E. degree from Rose Polytechnic Institute in 1971, the M.S.E.E. from Rose-Hulman Institute of Technology in 1984, and the Ph.D. from the University of Dayton in 1987. $\mathrm{He}$ is a member of Sigma $\mathrm{Xi}$ and a senior member of IEEE.

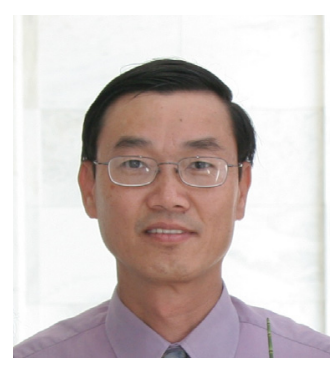

Dr. Seng Hong is a registered P.E., and the Program Manager at RF Countermeasures Assessment Lab, AFRL. Seng directs in-house R\&D activities, provides technical direction, and collaborates research efforts among DoD organization, defense industry, and academia. Prior coming to AFRL in 2002, Seng worked as contractor (AFEWES, Hangar 4F), and brings 16 years of hardware design and simulation experience related to EW technology. This includes foreign threat system reverse engineering, microwave receiver/RF components characterization, and data collection. Dr. Hong is a member of Tau Beta Pi, Eta Kappa Nu, Association of Old Crows, and IEEE.

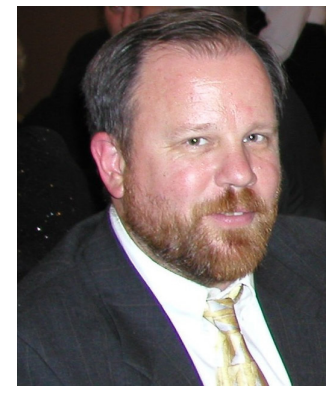

William Austin is the Technical Advisor for the Radio Frequency Sensor Assessment branch in the Sensors Directorate, Air Force Research Laboratory at Wright Patterson AFB, OH. In this position, he provides technical direction for Branch sensor assessment programs and leads the continuing development of the RF Countermeasures Assessment Facility and the Integrated Demonstrations and Applications Laboratory. Bill has worked in Electronic Warfare for over 20 years, primarily in EW simulation and testing. His experience runs across the entire EC test process, including establishing requirements for $\mathrm{EW}$ acquisition programs, performing EC simulations in hardware-in-the-loop laboratories, and conducting flight test evaluations.

John Glett received the B.S.E.E. degree from Cedarville University, Cedarville, $\mathrm{OH}$ in 2004, and the M.S.E.E. degree from Wright State University, Dayton, OH in 2007. He currently serves as an Electronics Engineer at the Air Force Research Lab Sensors Directorate, Wright-Patterson AFB, OH, where he was also stationed as a United States Air Force officer from 2004-2007. His research interests include high-speed digital and analog circuit design, RADAR systems, communications systems, and signal processing. 


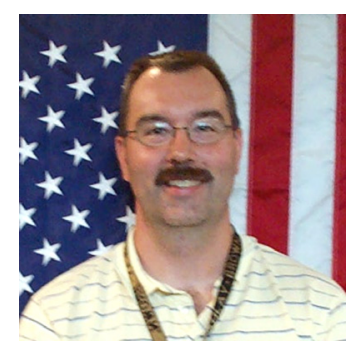

Mr. Mark Haenni, MacAulay Brown, began his career in 1987 at the Radar Laboratories of McDonnell Aircraft Company. He has held a variety of technical positions working with contractors for the Air Force laboratories. His work experience has included low observable antenna design, wideband digital receiver development and test, ECM test and assessment and ESM system integration. He holds the BSEE (1987) and the MSEE (1995) in Electrical Engineering, both from the University of Dayton, Dayton, $\mathrm{OH}$. He has been a member of Institute of Electrical and Electronics Engineers (IEEE) since 1996.

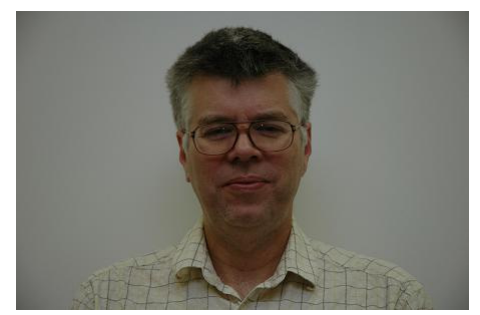

Mr. Gwynne Jones is an Electronics Engineer at the Air Force Research Lab, conducting research on radio frequency systems and antennas. He previously managed the testing and integration of avionics systems with the Aeronautical Systems Center (ASC) at Wright-Patterson AFB and a missile flight test program. He received a BSEE (1981) from the University of Virginia, Charlottesville, Virginia and a MSEE (1982) from the Air Force Institute of Technology at Wright-Patterson AFB, Ohio.

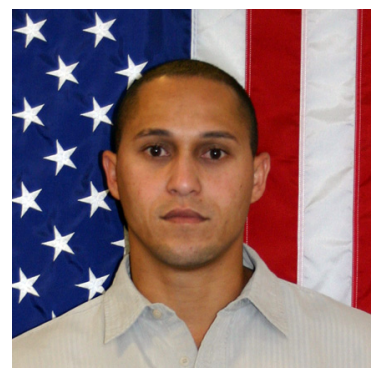

Mr. Rey Febo was born in New Jersey, USA on January 1978, and was raised in the island of Puerto Rico. He received the B.S. in electrical engineering from University of Puerto Rico, Mayagüez Campus, where he graduated with honors in 2007. He also served in the United States Marines Corps from May 1995 until December 2005, when he was released from active duty and resumed studies in his field, performing research in fuel cells. He is currently studying his M.S. degree in applied electromagnetics at the UPRM. His research interests are reconfigurable antennas with the application of MEMS switches. 